\title{
Ventral Hippocampal Neurons Are Shaped by Experience to Represent Behaviorally Relevant Contexts
}

\author{
Robert W. Komorowski, Carolyn G. Garcia, Alix Wilson, Shoai Hattori, Marc W. Howard, and Howard Eichenbaum \\ Center for Memory and Brain, Boston University, Boston, Massachusetts 02215
}

Memories can be recalled at different levels of resolution, from a detailed rendition of specific events within a single experience to a broad generalization across multiple related experiences. Here we provide evidence that neural representations reflecting the specificity or generality of memories are differentially represented along the dorsoventral axis of the CA3 area of the rat hippocampus. In dorsal CA3, neurons rapidly associate the identity of events with specific locations whereas, in more ventrally located CA3 regions, neurons gradually accumulate information across extended training to form representations that generalize across related events within a spatial context and distinguish events across contexts.

\section{Introduction}

The dorsal and ventral hippocampal regions have been differentiated along several lines of evidence. Anatomical projections to the dorsal and ventral hippocampus originate from largely nonoverlapping regions of the entorhinal cortex and terminate within separate regions of the dentate gyrus (DG; Dolorfo and Amaral, 1998). Lesions of the dorsal hippocampus impair spatial memory whereas lesions of the ventral hippocampus result in deficits in emotional and olfactory memory (Moser et al., 1993; 1995; Moser and Moser, 1998; Kjelstrup et al., 2002; Maren and Holt, 2004; Pothuizen et al., 2004; Rogers et al., 2006; Hunsaker and Kesner, 2008; Hunsaker et al., 2008). There are also major distinctions in genetic and molecular markers along the DV axis (Fanselow and Dong, 2010). By contrast to the large amount of data on anatomy and behavior, few studies have compared the firing properties of neurons along the dorsoventral (DV) axis, revealing only that, compared with dorsal hippocampal neurons, more ventral neurons have lower spatial specificity (Jung et al., 1994; Poucet et al., 1994; Royer et al., 2010) and greater place field size (Kjelstrup et al., 2008). The functional role of the gradient in place field size is currently unknown.

Here we explored differences in the firing patterns of neurons located along the DV axis in rats learning and performing a hippocampal-dependent task that requires the integration of object-reward associations with spatial cues that define one of two environmental contexts. Previously we have reported that the activity of individual dorsal hippocampal pyramidal neurons

Received Nov. 26, 2012; revised April 3, 2013; accepted April 4, 2013.

Author contributions: R.W.K. and H.E. designed research; R.W.K., C.G.G., A.W., and S.H. performed research; R.W.K. and M.W.H. analyzed data; R.W.K. and H.E. wrote the paper.

This work is supported by National Institutes of Health (NIH)/National Institute of Mental Health (NIMH) MH 051570 and Silvio 0. Conter Center Grant NIH/NIMH MH094263. We thank Andrew Bogaard for assistance with animal tracking data. Shea Gillet and Lauren Keller assisted with animal training and behavioral analyses.

The authors declare no competing financial interests.

Correspondence should be addressed to Howard Eichenbaum, Center for Memory and Brain, Boston University, 2 Cummington Street, Room 109, Boston MA 02215. E-mail: hbe@bu.edu.

DOI:10.1523/JNEUROSCI.5458-12.2013

Copyright $\odot 2013$ the authors $\quad 0270-6474 / 13 / 338079-09 \$ 15.00 / 0$ robustly encodes the sampling of specific objects at particular spatial locations within each spatial context, and the development of object-location representations parallels initial rapid learning of the context-dependent object associations (Komorowski et al., 2009; Tort and Komorowski et al., 2009). Now we report that CA3 neurons successively more ventral on the DV axis increasingly generalize among object sampling events that involve specific objects and locations within each spatial context, while distinguishing behavioral events that occur in different spatial contexts. Furthermore, ventral neurons gradually develop this property, eventually outperforming dorsal hippocampal neurons in linking behavioral events by context. These findings suggest that the ventral hippocampus integrates related events within a context across broad spatial and temporal scales.

\section{Materials and Methods}

Subjects. All animals were single housed and maintained on a $12 \mathrm{~h}$ light/ dark cycle (lights on 8:00 A.M. to 8:00 P.M.). Behavioral training and testing were conducted during the light phase. Animals were kept at $\sim 85 \%$ of their free-feeding body weight and had free access to water in the home cage. Procedures were conducted according to the requirements set by the National Institutes of Health and Boston University Institutional Animal Care and Use Committee.

Behavioral training. For electrophysiology, 11 male Long-Evans rats (400-600 g; Charles River) were trained on the context-dependent object association task as previously reported (Komorowski et al., 2009). After the animals were initially shaped to dig in $10 \mathrm{~cm}$ tall, $11 \mathrm{~cm}$ wide terra cotta pots filled with common playground sand for one-quarter Kellog's Froot Loop pieces they were trained on a simple discrimination between two pots filled with sand and scented with different oil fragrances placed side by side in the home cage. After achieving a performance criterion of 8 correct out of 10 consecutive trials within a single session, rats moved onto the next stage of training where they were allowed to forage for food scattered on the floor of the apparatus where training on the context-guided object association task took place (Fig. 1).

They next day commenced training on the context-dependent objectassociation task (Fig. 1). The apparatus involved two $37 \times 37 \mathrm{~cm}$ environments, decorated with different visual cues and operationally defined here as Context A and Context B, connected by a central alley that allowed the rat to shuttle between them. At the outset of each trial, the 


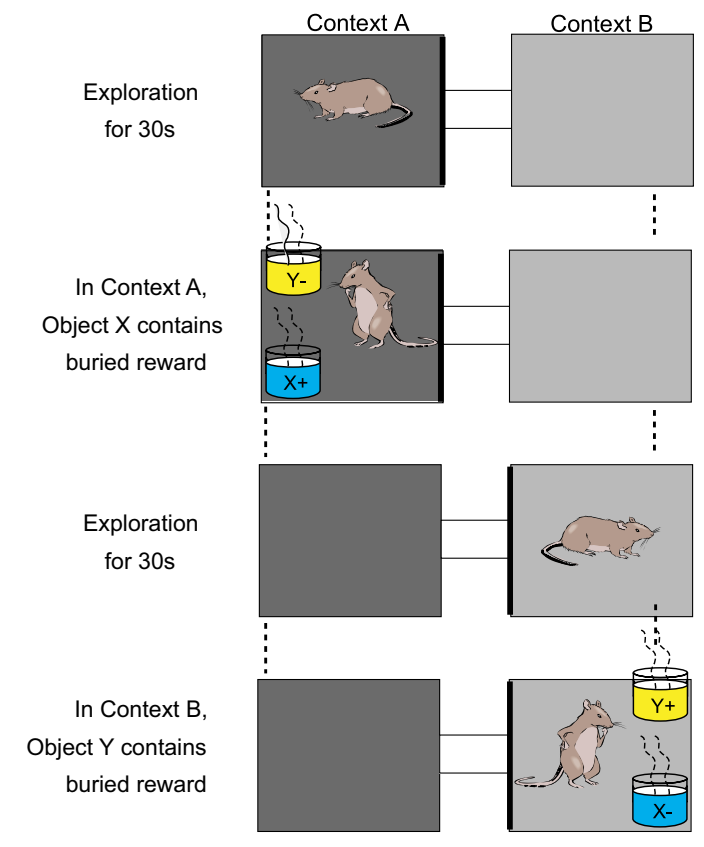

Figure 1. Context-guided object-association task. Successive context exploration and object sampling periods of two example trials. " +" indicates rewarded object and " -" indicates nonrewarded object in each context.

animal was permitted to explore one of the spatial contexts for $30 \mathrm{~s}$ (the context exploration period). Then the rat was presented with two terra cotta pots each scented with a different odor and filled with a different digging media and placed in the distant corners of the spatial context defined here as "locations" (the object sampling period). Objects appeared in each location equally often, but never in the same location on more than three consecutive trials. In Context A, Object X contained a Froot Loop reward, whereas in Context B, Object Y contained the reward. Digging in the correct pot uncovered the buried reward, but digging in the incorrect pot resulted in the removal of both pots and a $5 \mathrm{~s}$ time-out. To ensure that the animal did not simply learn to alternate choices of Objects X and Y, two successive trials were presented within the same spatial context every 10 trials on average. Initial training on this first problem required $3-5$ d of 80 trials per day until performance reached at least $70 \%$ in concurrent 10 trial blocks within each context.

Surgery. After reaching the performance criterion, rats were implanted with $12-18$ tetrodes at $4.96-6.28 \mathrm{~mm}$ posterior and $3.5-5.2 \mathrm{~mm}$ lateral to bregma. The headstage contained 12-18 independently movable tetrodes aimed at CA1 and CA3; however, only data from the CA3 region are reported. Each tetrode was composed of four $12.5 \mu \mathrm{m}$ nichrome wires with the tips plated with gold to bring the impedance to $200 \mathrm{k} \Omega$ at $1 \mathrm{kHz}$.

Recordings. Animals recovered for 7-10 d, after which the tetrodes were moved down slowly over the course of 1-2 weeks, until the tips reached the pyramidal cell layer and the animal's performance had again met criterion level on the initial context-guided object association problem. Tetrode locations were estimated in vivo using driver turn counts. During all recording sessions, spike activity was amplified $(10,000 \times)$, filtered $(600-6000 \mathrm{~Hz})$, and saved for off-line analysis using the software Spike (written by Loren Frank, University of California, San Francisco). Clusters of single-unit activity were isolated off-line and determined to be stable pyramidal units using various 3D projections (spike peak, valley, principal components, and time stamps) provided by Offline Sorter (Plexon). In addition, behavior was recorded with digital video (30 frames/s) that was synchronized with the acquisition of neural data and the animal's location was tracked with two LEDs located on the recording headstages.

Postsurgical training. Once the tetrodes were in the desired locations, recordings were taken as the rats continued to perform the initial context-dependent object association problem. Overtraining sessions were defined as sessions where the animal's performance had exceeded $80 \%$ for at least three consecutive prior testing days. Following these sessions, the animal was introduced to a novel environment with the same general configuration but with new flooring and new wallpaper defining each spatial context. After 15 min of exposure to this environment, the next day we began testing the animal on a second contextdependent object association problem using pots with new scented oils and digging media. Subsequent overtraining session recordings were taken on the second problem after the animal had again reached the criterion of $80 \%$ correct performance for at least three consecutive testing days.

Analysis. Cells from each tetrode were analyzed from only one learning session and one overtraining session to avoid the inclusion of the same neuron more than once in the each type of session. Separate analyses were performed on the context exploration period, when the rat explored the spatial context for $30 \mathrm{~s}$ without the presence of the objects, and on the object sampling period when the rat approached and investigated the two object stimuli; the onset and offset of stimulus sampling was defined manually from the video and imported to MATLAB for subsequent data analyses. Within each object sampling period, the onset of each object sampling event was defined as the time between when the animal's nose reached the rim of the pot and either when the animal began to dig, or removed its nose from the pot. There were often multiple object sampling events within each overall object sampling period.

All presumptive principal cells included in the analysis had a mean firing rate $<10 \mathrm{~Hz}$ per session and $<5 \mathrm{~Hz} /$ per pixel, and the place field covered $<80 \%$ of the maze area during spatial context exploration. Spatial firing rate maps for the context exploration period were estimated using the total number of spikes that occurred when the rat, with a velocity $>5 \mathrm{~cm} / \mathrm{s}$, was at a given location $(2 \mathrm{~cm} \times 2 \mathrm{~cm}$ bins $)$ divided by the total time spent in that bin and smoothed by the mean for all bins within $5 \mathrm{~cm}$, weighted by a 2D Gaussian kernel. A place field was defined as any contiguous set of at least 50 pixels with a firing rate $>20 \%$ of the peak firing rate for a given cell. Place field size was quantified as a percentage calculated as the number of bins within the field divided by all bins visited within the environment. Spatial firing rate maps for the object sampling period were calculated in a similar manner for the combination of all object sampling events within each object sampling period, except that the minimum size of the field was 25 adjacent pixels, as the area sampled was much smaller than that during context exploration.

Measures of selectivity used firing rates converted to $z$-scores calculated as the difference between a particular condition and the average firing rate across all stimulus samples, divided by the SD. Location selectivity during object sampling was measured as the difference between the average $z$-score firing rate at the preferred location (defined as the location with highest firing rate) and the alternate location within the same spatial context. Context selectivity during object sampling was measured as the difference in average $z$-score firing rates between Context $\mathrm{A}$ and Context B. Only cells that had an average firing rate of at least $0.1 \mathrm{~Hz}$ across all object sampling events were included.

Analyses of neural ensemble activity during object sampling were performed by creating vectors of normalized $z$-score firing rates during each object sampling event with a length of $n$ corresponding to the number of presumptive pyramidal units active during any given object sampling event. Ensembles consisted of at least two cells with a mean firing rate across all object samples of $>0.1 \mathrm{~Hz}$. The ensemble similarity between a pair of events ( $x$ and $y$ ) was measured as the cosine of the angle between them as plotted in $n$-dimensional space using the following formula:

$$
\cos (x, y)=\frac{x \cdot y}{\|x\|\|y\|}
$$

The similarity measure can range in value between -1 , where the vectors are completely opposite from one another, to 1 , where the vectors are identical; 0 indicates no relationship. Thus a cosine similarity of 1 reflects identical firing rates across all neurons within the ensemble between a pair of events. Measures of response reliability to a stimulus have been used as a measure of information coding (Bair and Koch, 1996; de Ruyter van Steveninck et al., 1997; DeWeese et al., 2003). We adapted this novel 


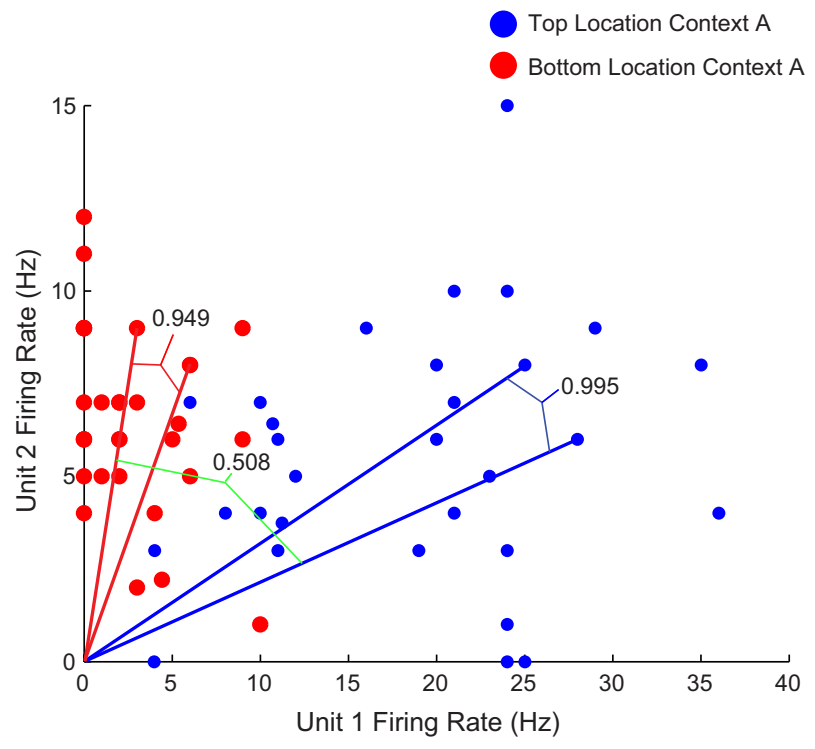

Figure 2. Ensemble cosine similarity analysis. Firing rates during object sampling events of units 1 and 2 , a subset of the total ensemble recorded in an animal during a learning session, are plotted with respect to the $x$-and $y$-axes. All events occurring at the top location within Context $A$ are in blue and all events at the bottom location are plotted in red. For simplicity, this figure uses raw firing rates; however, subsequent analyses used $z$-score transformations of the firing rate (see Materials and Methods).

measure of response reliability to quantify ensemble coding for a particular dimension (e.g., location, object, context, object/location) by taking the difference between the average ensemble cosine similarity for all object sampling event pairs of the same condition and the ensemble similarity of all object sampling event pairs of the opposite condition.

Since the number of dimensions is equal to the number of neurons in the ensemble, it is difficult to graphically illustrate multidimensional ensemble firing rate vectors. However, Figure 2 illustrates an example of ensemble coding for location using the smallest possible ensemble, 2 units, comparing firing at the top and bottom locations within Context A. Highlighted are example cosine values of the angle between a pair of "blue dot" events at the top location of Context A (0.995) and a single pair of "red dot" events that occurred at the bottom of Context A (0.949). These high cosine similarity values indicate that the ensemble response to these event pairs was very reliable. To obtain the cosine similarity for events at the same location (object sampling event pairs of the same condition), we calculate the cosines for all possible comparisons between pairs of events that occurred at the top of Context A (blue dots to blue dots) and all combinations of event pairs at the bottom location of Context A (red dots to red dots), to obtain an average cosine measure for all object sampling events occurring at the same location of 0.896 .

To measure the average cosine similarity between all pairs of object sampling events that occur at different locations (object sampling event pairs of the opposite conditions), we simply calculate the cosine of the angle between all combinations of events that occurred in the top and bottom of Context A, or red dots to blue dots. As shown by the green bracket, the cosine similarity for these events tends to be much lower ( 0.508 in the example) as there is a large difference in the firing rates of the unit pair between different locations. The average cosine measure then for all possible event pairs at different locations for this example is 0.574 .

Thus the measure of ensemble coding for location for this dataset is simply the difference between the average cosine similarity between all event pairs at the same location (0.896) and the average cosine similarity between event pairs at different locations (0.574), which is 0.322 .

Significant changes in ensemble coding were identified within a session using repeated measure ANOVAs, and across learning and overtraining sessions using independent sample ANOVAs. All statistics were performed using SPSS (IBM).
Histology and reconstruction of recording location. At the end of data collection, animals were anesthetized using isoflurane gas after which a $25 \mu \mathrm{A}$ current for $20 \mathrm{~s}$ was passed through each tetrode immediately before perfusion to create a lesion in the brain at the tip of each electrode. To quantify recording depth within hippocampus, all recording locations were plotted on a 2D unfolded map of the CA3 subregions of the hippocampus and depth measurements were taken relative to the dorsal CA2/3 border (Swanson et al., 1978). Dorsal, intermediate, and ventral hippocampal regions were assigned using previous identifications based upon genetic and anatomical profiles (Fanselow and Dong, 2010). Dorsal CA3 was defined as between 2 and $4 \mathrm{~mm}$ away from the dorsal CA2/3 border, intermediate CA3 was defined as between 4 and $7 \mathrm{~mm}$ away from the dorsal CA2 $/ 3$ border, and ventral CA3 was defined as $>7 \mathrm{~mm}$ away from the dorsal CA $2 / 3$ border.

Lesion experiment. In a separate group of 20 animals (300-400 g), rats were trained for 20 trials per day on the context-dependent objectassociation task until they reached the performance criterion described above. After which, they were anesthetized using isoflurane gas and held in a stereotaxic frame (David Kopf Instruments). Half of the animals then received infusions of a $0.9 \%$ sterile saline solution and the other half were infused with NMDA (Sigma) dissolved in $0.9 \%$ sterile saline solution (20 $\mathrm{mg} / \mathrm{ml}$ ) at seven sites in the hippocampus within each hemisphere (Fig. $3 A$ ). Following $7 \mathrm{~d}$ of recovery, these animals were retested for $5 \mathrm{~d}, 20$ trials per day, on the context-dependent object association task acquired before surgery.

\section{Results}

\section{Lesion experiment}

One control rat died during surgery and one hippocampal lesioned animal was excluded because of damage that extended into perirhinal cortex. Thus, there were nine controls and nine hippocampal lesioned animals. The extent and location of the lesion was evaluated at five coronal sections taken from locations ranging between $-2.16 \mathrm{~mm}$ to $-6.36 \mathrm{~mm}$ anteroposterior (Fig. $3 B$ ). On average, $79.2 \%$ (range $48-89 \%$ ) of the hippocampus was damaged across these five sections. In the $5 \mathrm{~d}$ of testing after recovery, animals with hippocampal lesions performed significantly worse than saline control on the previously acquired context-dependent object association task (Fig. $3 C ; t_{(16)}=3.52, p=0.003$ ) and performance did not correlate with lesion size $(r=-0.0527, p=0.893)$. Thus, the hippocampus is necessary for memory of well learned contextdependent object associations.

\section{Behavior for electrophysiology}

Rats acquired the context-dependent object-reward associations within a single learning session (Fig. $4 ; F_{(8,80)}=15.728 ; p<0.001$; $n=11$ ), demonstrating significant above chance performance within $41-50$ trials $\left(t_{(10)}=3.963, p=0.003\right)$.

\section{Recording locations and firing rates of CA3 neurons}

The analyses of single neuron firing patterns described below are based on 229 CA3 neurons recorded during the learning session: 163 neurons in dorsal CA3, 35 in middle CA3, and 31 in ventral $\mathrm{CA} 3$, and on $167 \mathrm{CA} 3$ neurons recorded during overtraining there were 96 in dorsal CA3, 40 in middle CA3, and 31 in ventral CA3. Average and peak firing rates during object sampling did not differ across DV levels (Table 1; Kruskal-Wallis test $H=2.46$, $\mathrm{df}=2, p=0.292$; peak firing rate: Kruskal-Wallis test $H=0.584$, $\mathrm{df}=2, p=0.747$ ) or across training stages (all Mann-Whitney comparisons: $Z=$ range -1.18 to -0.228 , all $p$ 's $>=0.238$ ).

Ventral CA3 neuronal representations that distinguish spatial contexts develop with extended training

Here we report the activity patterns of CA3 pyramidal neurons along the DV axis (229 recorded in the learning session, 167 in 
extended training sessions). First, we examined spatial firing patterns during the context exploration periods and found that the size of place fields differed along the DV axis (Fig. 5A) and this gradient was apparent both during learning (Fig. 5B, light gray bars: Kruskal-Wallis test $H=$ 25.81 , $\mathrm{df}=2, p<0.001)$ and in overtraining (Fig. 5B, black bars: Kruskal-Wallis test, $H=23.19$, $\mathrm{df}=2, p<0.001)$. The same pattern of larger place fields in more ventral areas was observed during the object sampling periods (Fig. $5 C$; learning, gray bars: $F_{(2,84)}=3.52, p=0.034$; overtraining, black bars: $\left.F_{(2,55)}=3.65, p=0.033\right)$. These results are consistent with a previous report that place field size increases along the DV axis (Kjelstrup et al., 2008).

Next, we examined firing patterns during the object sampling events (see Materials and Methods) of learning sessions and observed that many dorsal CA3 neurons fired only when objects were sampled at a particular location within one or both spatial contexts, whereas activity in ventral CA3 neurons more often poorly discriminated between multiple locations within and across spatial contexts (for example, Fig. 6A, upper left ventral CA3 cell). Dorsal CA3 neurons continued to exhibit locationspecific firing patterns in overtraining sessions (Fig. $6 B$, examples of dorsal CA3 cells). In contrast, extended training reshaped ventral CA3 activity, such that ventral neurons came to fire similarly between locations within a spatial context while maintaining differential firing patterns between contexts, as can be seen in all of the examples of ventral hippocampal cells in Figure $6 B$.

We quantitatively characterized the extent to which neuronal activity differentiated locations within a spatial context (location selectivity) and the extent to which they differentiated the two spatial contexts (context selectivity) during initial learning and overtraining, using normalized ( $z$-score) firing rates of neurons located across DV positions. Location selectivity (Fig. 6C), diminished at increasingly ventral positions (main effect of region: $F_{(2,138)}=4.02, p=0.02$ ), regardless of training condition (no main effect of training: $\left.F_{(1,138)}=0.511, p=0.476\right)$. In contrast to the pattern observed with location selectivity, context selectivity did not differ by DV region during initial learning (Fig. $6 D$, light gray; $\left.F_{(2,85)}=1.06, p=0.352\right)$ but did increase in ventral regions during overtraining (Fig. $6 D$, black; $F_{(2,53)}=3.24, p=0.047$ ). Thus single-unit context selectivity increased only within the ventral hippocampus upon extended training (overall interaction of training with recording region: $F_{(2,138)}=4.50, p=0.013$ ).

Can the observed differences in location and context selectivity be due to differences in place field size across the DV axis? To directly address this question, we combined cells across DV levels and correlated place field size with location and context selectivity measures. Place field size was not significantly correlated with location selectivity either in the learning session $(r=-0.040, p=0.710)$ or in overtraining $(r=-0.102, p=0.445)$. Therefore the larger place field sizes observed in ventral CA3 throughout testing (Fig. 5C) do not account for the observed lower location selectivity (Fig. 5C). Rather, as can be seen in the examples shown in Figure $5 A$, the larger ventral CA3 place fields usually extended only to areas adjacent to the location of one object and seldom to the location of the other object.

Place field size is significantly correlated with context selectivity in both the learning session $(r=0.223, p=0.038)$ and in overtrain-

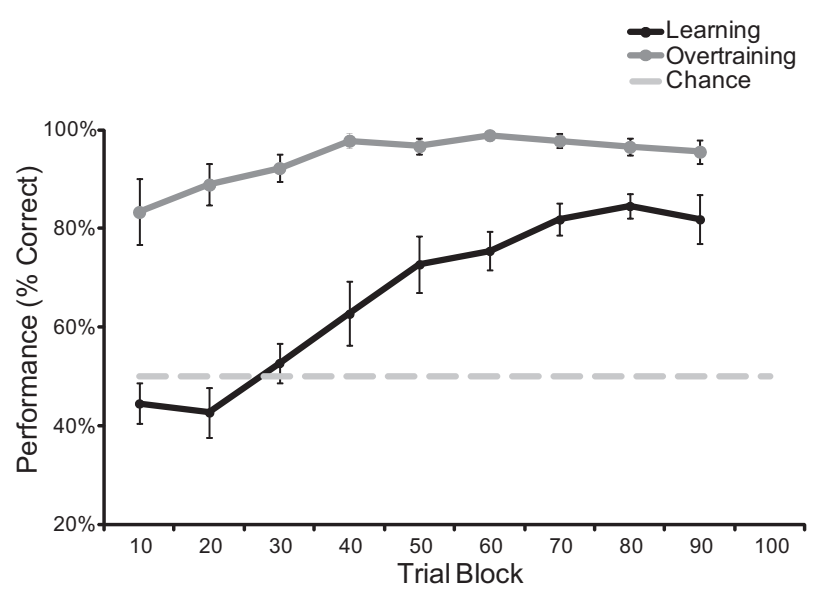

Figure 4. Performance in learning and overtraining. The learning curve represents the average performance of 11 rats within bins of 10 trials. The extended training curve reflects performance of 9 of the same 11 rats across nine sessions. Performance did increase significantly during overtraining (repeated-measures ANOVA: $F_{(8,64)}=3.217 ; p=0.004 ; n=9$ ), but was significantly different from learning (repeated-measures ANOVA interaction: $F_{(8,144)}=5.687$; $p<0.001)$. Error bars indicate $1 \pm$ SEM.

ing $(r=0.30, p=0.003)$. However, while ventral CA3 place fields were larger during learning (Fig. $5 \mathrm{C}$ ), at this stage dorsal and ventral CA3 neurons did not significantly differ in context selectivity; indeed the average context selectivity score for ventral CA3 neurons was slightly lower than that for dorsal CA3 neurons (Fig. 6D). Also, while ventral hippocampal neurons developed stronger context selectivity with overtraining (Fig. $6 D$ ), there was no significant change in the size of ventral hippocampal place fields between learning and overtraining sessions; indeed the average place field size of ventral CA3 cells was slightly lower in overtraining than in the learning session (Fig. 5C). Therefore the improvement in context discrimination during extended training in ventral CA3 neurons was due to the reshaping of their place fields to extend across locations within a spatial context while restricted to one of the spatial contexts, rather than an overall increase in place field size (Fig. 6A,B).

Combining these observations, neurons within the ventral CA3 gradually developed a representation that ultimately excelled at generalizing across object sampling events with comple- 
Table 1. Peak and average firing rates during object sampling

\begin{tabular}{|c|c|c|c|c|c|c|}
\hline & \multicolumn{3}{|l|}{ Learning } & \multicolumn{3}{|l|}{ Overtraining } \\
\hline & Dorsal & Middle & Ventral & Dorsal & Middle & Ventral \\
\hline Peak firing rate & $7.0(2.0-36.0)$ & $8.2(2.0-23.0)$ & $4.6(2.0-27.0)$ & $6.9(2.0-29.9)$ & $6.3(3.0-29.0)$ & $7.0(3.0-31.0)$ \\
\hline Average firing rate & $0.60(0.11-12.60)$ & $0.80(0.11-2.80)$ & $0.40(0.11-1.70)$ & $0.50(0.10-9.70)$ & $0.70(0.14-5.94)$ & $0.40(0.12-8.50)$ \\
\hline
\end{tabular}

The median (and range) for peak and average firing rates recorded during object sampling events.

A

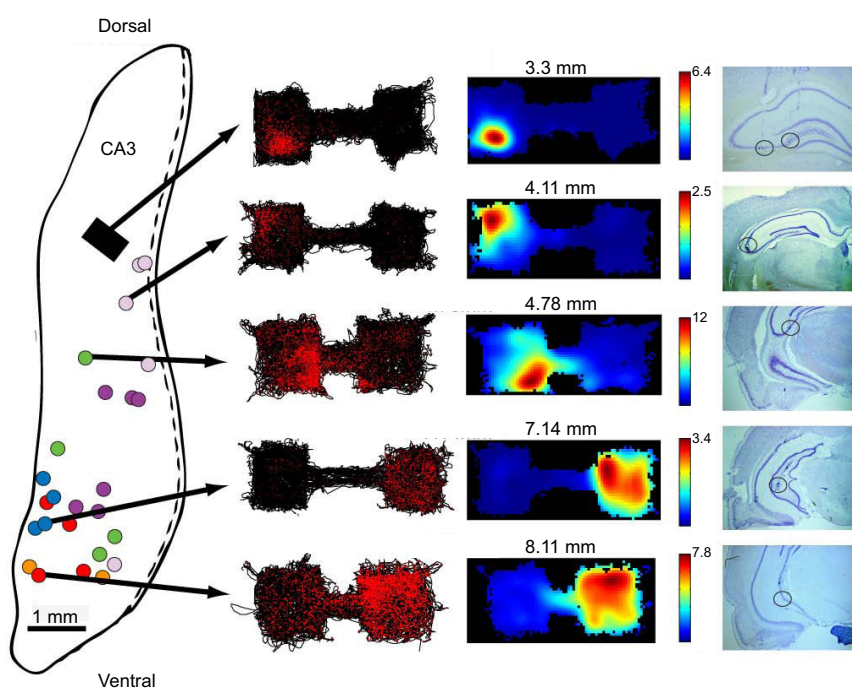

B



C

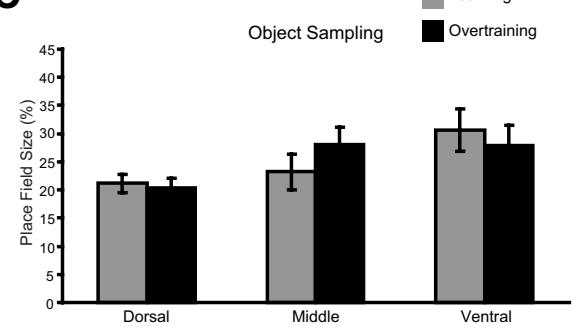

Figure 5. Place field sizes in learning and with overtraining. $\boldsymbol{A}$, Tetrode locations and example spatial firing patterns and place fields. Tetrodes in the five rats from our previous study (Komorowski et al., 2009) were often too close together to determine their exact location, and therefore are represented by gray boxes. Tetrode locations for the six new rats are depicted in colored circles with locations for each rat in the same color on a 2D flattened map of hippocampus (Swanson et al., 1978). To the right shows the spatial firing plots for five cells selected from different recording depths in rats during the extended training condition. On the left is the rat's path (black lines) along with red dots indicating the location where spikes occurred. To the right are normalized spatial firing rate maps generated from the spiking data. Histological sections show the tetrode location for each example cell. $\boldsymbol{B}$, Place field size measured during the context exploration period. Error bars indicate average place field size ( $\pm \mathrm{SE})$ as a percentage of the context for neurons in dorsal, middle, and ventral CA3. C, Same place field measures for the object sampling period.

mentary reward values at different locations within a spatial context (e.g., objects $\mathrm{X}^{+}$and $\mathrm{Y}^{-}$in Context $\mathrm{A}$ ) and also eventually excelled at distinguishing object sampling events that occurred within spatial contexts of differential significance (e.g., object $\mathrm{X}^{+}$in Context A vs object $\mathrm{X}^{-}$in Context $\mathrm{B}$ ).

At the level of neural ensembles, ventral CA3 slowly develops representations that generalize across object sampling events within a spatial context, whereas dorsal $\mathrm{CA} 3$ rapidly develops specific location and object-context conjunctive representations

Next we performed analyses on neural ensembles combined at previously identified dorsal, intermediate, and ventral areas within CA3 (Fanselow and Dong, 2010), to characterize the abil- ity of different DV areas to code for object sampling events in parameters relevant to task performance. The ensemble analyses compared the extent to which neuronal populations in each area activated consistently during object sampling events that match in identity while at the same time distinguishing between events that differ in identity within the same dimension. Previous studies have characterized ensemble coding of stimulus dimensions by ensemble vector cosines across repeated presentations of a stimulus (Bair and Koch, 1996; de Ruyter van Steveninck et al., 1997; DeWeese et al., 2003). We adapted this approach to object, location, and context coding, as well as conjunctions of object location for all object sampling events (see Materials and Methods and Fig. 2). For each measure we quantified ensemble coding as the difference between the average cosine similarity for all pairs of object sampling events at the same value of one of those dimensions versus the cosine similarities at different values of the same dimension. For example, to quantify ensemble coding of location within a spatial context, we measured the difference between the average cosine similarity for all pairs of object sampling events at the same location versus all pairs of object sampling events at different locations within the same spatial context. Ensemble sizes were 6-16 $(n=9)$ in dorsal CA3, 3-6 $(n=5)$ in middle CA3, and $2-5(n=8)$ in ventral CA3.

As was expected, throughout the learning session dorsal ensembles strongly coded for the locations of object sampling events, and location coding decreased in a graded manner in intermediate and ventral hippocampus (Fig. $7 A$; main between-subjects effect of region: $F_{(2,8)}=6.37, p=0.022$ ), but was not influenced by acquisition of the task at any recording location (repeated-measures 30 trial bins: $F_{(2,16)}=0.88, p=$ 0.432 and interaction of trial with recording region $F_{(4,16)}=0.80$, $p=0.542$ ). However, within the initial learning session, dorsal ensembles evolved to distinguish pairs of object sampling events involving the same objects versus pairs of events involving different objects (Fig. $7 B ; F_{(2,8)}=4.89, p=0.041$; black line, performance: $\left.F_{(2,20)}=84.808, p<0.001\right)$, thus reflecting coding for the identity of objects. Further examination revealed that ensemble object coding was predominantly observed for object sampling events that were presented at the same location (Fig. $7 C ; F_{(2,8)}=$ $6.27, p=0.023)$. In contrast, intermediate and ventral hippocampal 
ensembles did not significantly distinguish objects or object-location combinations during learning (Fig. $7 B, C$; one-sample $t$ test vs 0 all $p>0.05$ ), nor were they modified during learning (repeated-measures 30 trial bins $p=>0.05$ ).

Subsequently, we explored whether the strength of object coding observed in the dorsal CA3 reflected task performance. Using the same ensemble measure as described above, we separated location, object, and object/location into sampling events occurring on correct versus incorrect trials. Interestingly, while ensemble activity representing object location was not influenced by trial outcome (Fig. $7 D$, yellow; $\left.F_{(1,8)}=0.04, p=0.953\right)$, there was a trend toward a decreased ensemble similarity related to objects on incorrect trials (Fig. $7 D$, blue; $F_{(1,8)}=5.01, p=0.056$ ). This trend reached significance when we compared the ability of ensembles to reflect the identity of objects encountered at the same location on correct versus incorrect trials (Fig. $7 D$, green; $F_{(1,8)}=9.53$, $p=0.015)$. Thus object coding in the dorsal hippocampus reflected the performance of the rat during learning, and performance was poor when dorsal ensembles failed to reflect the both the location and identity of an object sampling event.

Additional analyses quantified ensemble activity at different DV levels between learning and overtraining sessions. Ensemble coding of spatial context was measured by subtracting the average cosine similarity for all pairs of object sampling events in different spatial contexts (measuring the ability to differentiate contexts) from the average cosine similarity between all pairs of events at different locations in the same spatial context (measuring the ability to generalize across locations). High positive values indicate the ability of the ensemble to both generalize across locations and at the same time differentiate contexts. Most strikingly, in contrast to the superior and rapidly acquired ability of dorsal CA3 ensembles in differentiating locations within a spatial context and object-location conjunctions, ventral CA3 areas were ultimately superior at linking between object sampling events occurring at different locations within the same environmental context while maintaining context selectivity, in a profile that increased from dorsal to ventral CA3 only with extended training experience (Fig. $8 A$; effect of recording regions during learning: $F_{(2,8)}=1.34, p=0.315$; overtraining: $F_{(2,8)}=29.51$, $p<0.001$; interaction of recording region with training: $\left.F_{(2,16)}=13.85, p<0.001\right)$. Note that the increase in ensemble coding of spatial context cannot be attributed to the larger place fields in ventral hippocampus, because place fields did not change size with extended training (Fig. $5 C$ ).

We then compared these findings with ensemble coding of location during extended training. As described above for the analyses of learning sessions, location coding was measured by subtracting the average cosine similarity for all pairs of object sampling events at different locations within the same spatial context from the average cosine similarity for all pairs of object sampling events occurring at the same location. Thus, a high
B
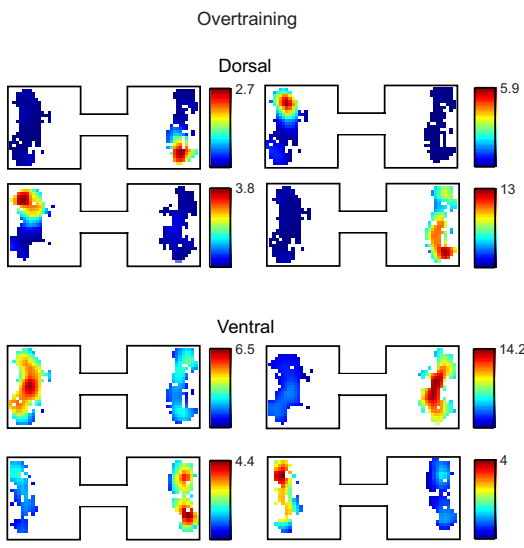

D

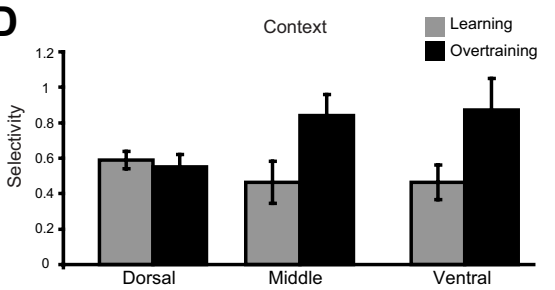

Figure 6. Location and context selectivity during object sampling. Normalized spatial firing rate maps for the object sampling period in the learning session $(\boldsymbol{A})$ and in overtraining sessions $(\boldsymbol{B})$. Each set of plots represents the spatial firing patterns of four location with the maximum firing rate) and the other location, within the same context. $\boldsymbol{D}$, Average ( \pm SE) single cell context selectivity measured as the difference in z-score normalized firing rate between a cell's preferred object context (context with the maximum firing rate across both locations) and the other context.
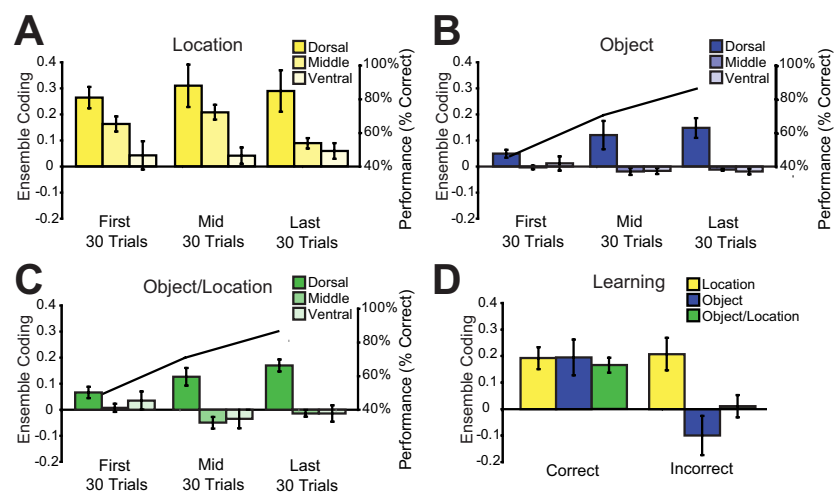

Figure 7. Object sampling ensemble coding within learning sessions. $\boldsymbol{A}$, Average ( $\pm \mathrm{SE}$ ) location coding across all rats in dorsal, intermediate, and ventral CA3 is shown in the first, middle, and last 30 trials of the learning session. The middle 30 trials were centered on the trial block in which the rat achieved our learning criterion (see Materials and Methods). B, C, Average ( \pm SE) Object and Object/Location coding in the same conditions as Fig 6 A. Black line represents average performance across all rats within each 30 trial block. $D$, Comparison of Location, Object, and Object/Location coding on trials in which the animal eventually made a correct versus incorrect choice. Averages here are not binned and reflect all trials within the learning session.

positive value indicates a strong tendency for the ensemble to link together events at a single location within the spatial context while still having enough spatial resolution to differentiate between locations within a spatial context. This measure revealed that the improvement in spatial representation within ventral CA3 ensembles between learning and overtraining sessions was not accompanied by a similar improvement for ensemble representations of object locations (Fig. 8B). Extended training failed 
A
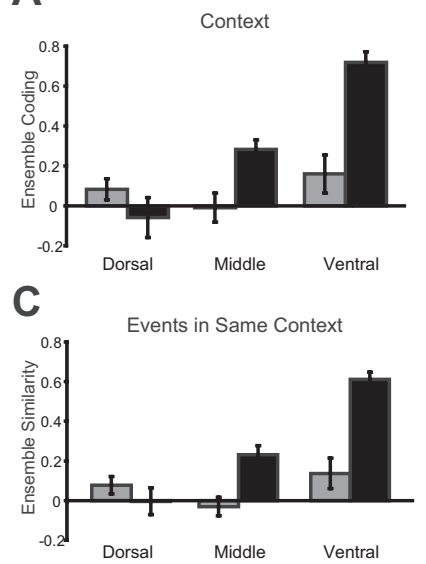

B

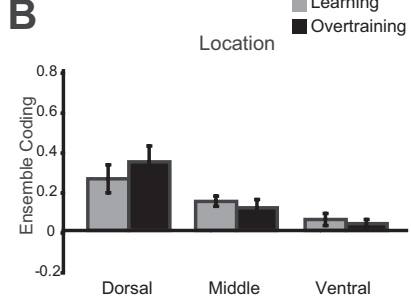

D

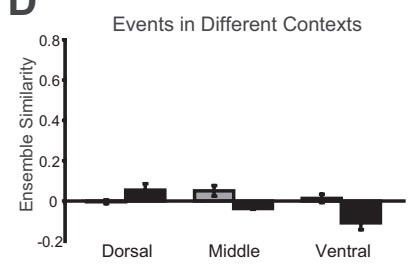

Figure 8. Object sampling ensemble coding comparing learning and overtraining sessions. $\boldsymbol{A}$, Average ( \pm SE) Context coding in dorsal, intermediate, and ventral hippocampus is shown in learning and in overtraining sessions. $\boldsymbol{B}$, Same plots, now for location coding. $\boldsymbol{C}, \boldsymbol{D}$, Breakdown of context coding results depicted in Figure $7 A$ into its components of average ensemble coding between pairs of object sampling events within the same context, but at different locations ( $\boldsymbol{C}$ and average ( $\pm \mathrm{SE}$ ) ensemble coding between pairs of object sampling events in different contexts.

to impact location coding within dorsal CA3, nor was it able to induce coding for individual object locations in the ventral CA3 (interaction of recording region with training: $F_{(2,16)}=1.91, p=$ 0.277 ). Notably, these improvements in the representations of spatial context did not appear within a recording session (repeated-measures interaction of 30 trial training bin with recording region in learning: $F_{(4,16)}=0.252, p=0.904$, and overtraining: $\left.F_{(2,16)}=1.91, p=0.277\right)$ nor did they reflect single trial performance (interaction of trial performance with recording region in learning: $\left.F_{(4,16)}=0.543, p=0.706\right)$. Thus extended experience slowly drove the formation of context selective representations that increased in strength moving down the long axis of the hippocampus.

A breakdown of the analyses of ensemble coding of spatial context revealed that the increase in context-selective representations in overtraining sessions was driven both by an increase in ensemble activity that generalized object sampling events across locations within a spatial context (Fig. $8 C$; effect of recording regions during learning: $F_{(2,8)}=1.32, p=0.321$; overtraining: $F_{(2,8)}=37.18, p<0.001$; interaction of recording region with training: $\left.F_{(2,16)}=13.48, p<0.001\right)$, and a complementary, albeit more modest, increase in the ability of ensembles to differentiate object sampling events between the two spatial contexts (Fig. $8 D$; effect of recording regions during learning: $F_{(2,8)}=2.21, p=0.172$; overtraining: $F_{(2,8)}=8.10, p=0.012$; interaction of recording region with training: $\left.F_{(2,16)}=8.80, p=0.003\right)$. Therefore, in contrast to dorsal CA3 ensembles, ventral CA3 ensembles were able to code for spatial context by generalizing object sampling events across locations within a spatial context, while also creating representations that differentiated events between spatial contexts (three-way interaction of training by recording region by Same/Different Context Cosines; $\left.F_{(2,32)}=20.40, p<0.001\right)$.

Notably, the differences in ensemble coding scores between hippocampal areas were not due to the differences in the sizes of ensembles used in those comparisons, as shown in two additional analyses. One of these analyses examined the data from the two animals with the largest number of recorded cells within an ensemble and measured cosine similarity by randomly selecting cells from the entire ensemble, ranging from 2 (the smallest pos- sible ensemble) up to the all of the cells recorded ( $n=16$ for both). Ensemble coding for location, as well as object/location conjunctions varies very little across ensemble sizes (Rat 3 Location: $F_{(14,182)}=0.390, p=0.976$; Object/Location: $F_{(14,182)}=$ $0.264, p=0.997$; Rat 11 Location: $F_{(14,182)}=0.523, p=0.876$; Object/Location: $\left.F_{(14,182)}=0.713, p=0.760\right)$. In another analysis we compared the average ensemble coding scores for the mean of 100 randomly selected four-cell dorsal hippocampal ensembles to the full ensemble scores and found no difference in scores during learning (location: $t_{(8)}=-0.06, p=0.953$; object: $t_{(8)}=$ $-0.126, p=0.903$; context: $t_{(8)}=-0.448, p=0.666$; object/ location: $t_{(8)}=0.315, p=0.761$ ) or during overtraining (location: $t_{(6)}=-0.482, p=0.647$; object: $t_{(6)}=0.257, p=0.806$; context: $t_{(6)}=-0.205, p=0844$; object/location: $t_{(6)}=-0.603$, $p=0.569)$. In addition, when four-cell ensemble coding scores were compared with the scores generated from ventral hippocampal ensembles of the same average size, the same pattern of results as that for full dorsal ensembles was observed: location and object/location coding were higher in dorsal hippocampus in both in learning (location: $t_{(7)}=2.543, p=0.038$; object/location: $t_{(7)}=3.332, p=0.013$ ) and overtraining (location: $t_{(6)}=$ $3.380, p=0.015$; object/location: $\left.t_{(6)}=4.971, p=0.003\right)$. Also, in agreement with our findings including all cells, ensemble context coding did not differ between dorsal and ventral ensembles in learning $\left(t_{(7)}=-0.112, p=0.914\right)$, but did after overtraining $\left(t_{(6)}=-7.41, p<0.001\right)$. These findings show that the main results are replicated using a subset of the dorsal data of similar size to the ventral data, so the differences in ensemble size cannot explain the pattern of results on differential coding along the DV axis.

The combined results of these ensemble analyses reveal a double dissociation of functional gradients along the DV axis, such that more dorsal CA3 ensembles excel in coding specific object sampling events and their locations associated with rapid initial learning whereas intermediate and ventral CA3 ensembles excel in linking events within a meaningful spatial context and distinguished events across spatial contexts in gradually developing generalized representations.

\section{Discussion}

The present findings indicate that experience in a task requiring the integration of information about events within meaningfully distinct spatial contexts can modify ventral hippocampal neuronal firing patterns. The decrease in the fidelity of spatial information with increasing place field size along the DV axis in the hippocampus has been well characterized (Jung et al., 1994; Poucet et al., 1994; Kjelstrup et al., 2008; Royer et al., 2010), but a functional role for these larger fields with respect to memory has only been suggested (Hasselmo, 2008). Here, we show that ventral hippocampal neurons not only have larger place fields but also represent information about behavioral events differently and these representations evolve with a different time course. Thus, whereas dorsal hippocampal neurons and neuronal ensembles rapidly developed representations of specific objects and locations of object sampling events in parallel with initial learning, ventral hippocampal neurons more gradually generalize across events within a meaningful spatial context while strongly distinguishing between meaningfully distinct spatial contexts. Although in this study we define context in terms of space, it is conceivable that notion of a functional context could extend to information about emotion or interoceptive cues (Hirsh, 1974; Fanselow and Dong, 2010). It would be of considerable interest to determine whether factors such as stress or emotion could uncover 
conditions under which ventral ensembles might be modified more quickly.

Importantly, while memory performance during learning has previously been related to dorsal hippocampal neuronal activity in a previous study (Komorowski et al., 2009), here we show that this activity is critical for performance on a single trial basis. We could not determine whether the more generalized representations within intermediate and ventral hippocampal areas are critical for memory performance because there were too few error trials to analyze when the rats were overtrained. However, this seems unlikely because ventral ensembles do not provide information about the identity of the objects, even with extended training. This finding is contrary to results of previous lesion studies indicating a role for ventral hippocampus in olfactory memory (Kesner et al., 2010, 2011) and the stimuli here are distinguished by olfactory qualities. Instead, the slow evolution of ventral hippocampal representations that are event general and context specific suggests that the ventral hippocampus extracts object and spatial information that is common between complementary and related experiences within the same context, as supported by lesion studies (de Hoz et al., 2003; Koh et al., 2009), which could reflect an interleaving of related memories during systems level consolidation (Tse et al., 2007). Ventral hippocampal representations may thus embody the "schema" for the set of object associations assigned within each environmental context, which can be further rapidly elaborated by later acquired associations in the same contexts (Tse et al., 2007).

The mechanisms by which the modification rate of neural ensembles differs along the DV axis is unclear; however, differences in neurogenesis in the input structure to CA3, the DG, may provide some insights. Recently it has been shown that newly born neurons in the DG are highly excitable (Espósito et al., 2005) and plastic (Ge et al., 2007) as they near the end of their maturation process and thus it has been argued they can respond to and be modified by experience most easily during this time in development (Aimone et al., 2009). Interestingly, newly born neurons in the ventral DG take longer to reach maturity than they do in dorsal DG and as a result new dorsal DG neurons spend less time in this highly plastic window than ventral DG neurons (Piatti et al., 2011; Snyder et al., 2012). The shorter window of elevated excitability and plasticity in dorsal DG would be ideal for the rapid association of new detailed information into the hippocampal network, which could then be further processed in dorsal CA3 to quickly influence behavior. Conversely, while the prolonged window of functional plasticity in ventral DG might introduce too much interference to be useful for the encoding of detailed information, it is ideal for providing neurons the time necessary to accumulate information and identify trends that develop across longer temporal intervals. This would contribute to the observed slower evolution of ventral CA3 ensembles. This rate of change may be ideal for tasks such as rule learning, which require the integration of information gatherer over repeated experiences.

Notably, it is the ventral subdivision of the hippocampus that sends direct inputs to the prefrontal cortex (Swanson, 1981), suggesting that a key role of the ventral hippocampus may be to send contextual information to the prefrontal cortex, which then can be used to bias the retrieval of specific memories appropriate to that context (Cohen et al., 1996; Miller, 1999). Within the framework of the current behavioral paradigm, context representations formed in the ventral hippocampus during the context exploration period might be sent to the prefrontal cortex, which subsequently applies a context-defined rule to guide retrieval of the appropriate specific memories that are represented in the dorsal hippocampus during the subsequent object sampling phase of the current trial. Such a scenario is consistent with the recent finding that inactivation of prefrontal cortex results in loss of object selectivity of dorsal hippocampal neurons in the same behavioral paradigm, such that dorsal hippocampal neurons normally acquired unique object-place associations, but after prefrontal activation, generated robust responses to both objects in the preferred place (Navawongse and Eichenbaum, 2013).

While the human literature comparing the loci of functional magnetic resonance imaging activations along the anterior-posterior axis of the hippocampus (analogous to ventral and dorsal hippocampus in rodents, respectively) has been mixed; some recent findings are quite consistent with the present results. Liang et al. (2013) reported that activation of the anterior hippocampus was generalized across different domains of event content, whereas activation of the posterior hippocampus discriminated information within specific scenes. Similarly, Awipi and Davachi (2008) found that the activation of the anterior hippocampus generalized across different categories of recollected content, whereas activation of the posterior hippocampus differentiated scene stimuli along with specific associated details. Also, Nadel et al. (2013) compared activations along the DV axis as subjects remembered either the general spatial context in which an experience occurred (remembering where a wedding occurred) or detailed spatial relations between events (remembering who was sitting on your left), and reported that remembering spatial context more greatly activated the anterior hippocampus whereas remembering detailed spatial relations more greatly activated the posterior hippocampus. These findings suggest that the human hippocampus also may coordinate representations of events at graded levels of specificity, similar to the observations at the neuronal level here.

Previous studies on the DV axis of the hippocampus have suggested either a unitary hippocampal function in mapping space across a range of scales (Hasselmo, 2008; Kjelstrup et al., 2008) or distinct DV subdivisions that selectively process spatial or nonspatial information (Moser et al., 1993, 1995; Bannerman et al., 2004; Fanselow and Dong, 2010). The present results incorporate aspects of both models. The dorsal hippocampus rapidly encodes related information about discrete events to form conjunctional representations containing high-resolution information about "what" and "where" for specific events. In contrast, ventral hippocampal areas gradually link related events across many experiences in the same context, while also strongly distinguishing meaningfully different contexts. It is important to note that these findings may be specific to CA3. Although we did notice similar types of coding in CA1 (not reported), it might be expected that information coding along the DV axis of CA3 may vary more gradually, as there are many longitudinal connections within CA3 that are not present in CA1 (Witter, 2007).

In conclusion, these results suggest that the hippocampus represents memories using continuous "topographic" mapping that ranges from representations of highly specific single events to representations of multiple events that are related by a distinct spatial context. Thus the entire hippocampus likely plays a unified function that supports encoding of memories at multiple scales of detail, a function that is critical for the successful recall of rich episodic memories.

\section{References}

Aimone JB, Wiles J, Gage FH (2009) Computational influence of adult neurogenesis on memory encoding. Neuron 61:187-202. CrossRef Medline

Awipi T, Davachi L (2008) Content-specific source encoding in the human medial temporal lobe. J Exp Psychol Learn Mem Cogn 34:769-779. CrossRef Medline

Bair W, Koch C (1996) Temporal precision of spike trains in extrastriate 
cortex of the behaving macaque monkey. Neural Comput 8:1185-1202. CrossRef Medline

Bannerman DM, Rawlins JN, McHugh SB, Deacon RM, Yee BK, Bast T, Zhang WN, Pothuizen HH, Feldon J (2004) Regional dissociations within the hippocampus-memory and anxiety. Neurosci Biobehav Rev 28:273-283. CrossRef Medline

Cohen JD, Braver TS, O'Reilly RC (1996) A computational approach to prefrontal cortex, cognitive control and schizophrenia: recent developments and current challenges. Philos Trans R Soc Lond B Biol Sci 351: 1515-1527. CrossRef Medline

de Ruyter van Steveninck RR, Lewen GD, Strong SP, Koberle R, Bialek W (1997) Reproducibility and variability in neural spike trains. Science 275: 1805-1807. CrossRef Medline

de Hoz L, Knox J, Morris RG (2003) Longitudinal axis of the hippocampus: both septal and temporal poles of the hippocampus support water maze spatial learning depending on the training protocol. Hippocampus 13: 587-603. CrossRef Medline

DeWeese MR, Wehr M, Zador AM (2003) Binary spiking in auditory cortex. J Neurosci 23:7940-7949. Medline

Dolorfo CL, Amaral DG (1998) Entorhinal cortex of the rat: topographic organization of the cells of origin of the perforant path projection to the dentate gyrus. J Comp Neurol 398:25-48. CrossRef Medline

Espósito MS, Piatti VC, Laplagne DA, Morgenstern NA, Ferrari CC, Pitossi FJ, Schinder AF (2005) Neuronal differentiation in the adult hippocampus recapitulates embryonic development. J Neurosci 25:10074-10086. CrossRef Medline

Fanselow MS, Dong HW (2010) Are the dorsal and ventral hippocampus functionally distinct structures? Neuron 65:7-19. CrossRef Medline

Ge S, Yang CH, Hsu KS, Ming GL, Song H (2007). A critical period for enhanced synaptic plasticity in newly generated neurons of the adult brain. Neuron 54: 559-566. CrossRef Medline

Hasselmo ME (2008) Neuroscience. The scale of experience. Science 321: 46-47. CrossRef Medline

Hirsh R (1974) The hippocampus and contextual retrieval of information from memory: a theory. Behav Biol 12:421-444. CrossRef Medline

Hunsaker MR, Kesner RP (2008) Dissociations across the dorsal-ventral axis of CA3 and CA1 for encoding and retrieval of contextual and auditory-cued fear. Neurobiol Learn Mem 89:61-69. CrossRef Medline

Hunsaker MR, Fieldsted PM, Rosenberg JS, Kesner RP (2008) Dissociating the roles of dorsal and ventral CA1 for the temporal processing of spatial locations, visual objects, and odors. Behav Neurosci 122:643-650. CrossRef Medline

Jung MW, Wiener SI, McNaughton BL (1994) Comparison of spatial firing characteristics of units in dorsal and ventral hippocampus of the rat. J Neurosci 14:7347-7356. Medline

Kesner RP, Hunsaker MR, Ziegler W (2010) The role of dorsal and ventral CA1 in memory for the temporal order of a sequence of odors. Neurobiol Learn Mem 93:111-116. CrossRef Medline

Kesner RP, Hunsaker MR, Ziegler W (2011) The role of dorsal and ventral hippocampus in olfactory working memory. Neurobiol Learn Mem 96: 361-366. Medline

Kjelstrup KB, Solstad T, Brun VH, Hafting T, Leutgeb S, Witter MP, Moser EI, Moser MB (2008) Finite scale of spatial representation in the hippocampus. Science 321:140-143. CrossRef Medline

Kjelstrup KG, Tuvnes FA, Steffenach HA, Murison R, Moser EI, Moser MB (2002) Reduced fear expression after lesions of the ventral hippocampus. Proc Natl Acad Sci U S A 99:10825-10830. CrossRef Medline

Koh MT, Wheeler DS, Gallagher M (2009) Hippocampal lesions interfere with long-trace taste aversion conditioning. Physiol Behav 98:103-107. CrossRef Medline
Komorowski RW, Manns JR, Eichenbaum H (2009) Robust conjunctive item-place coding by hippocampal neurons parallels learning what happens where. J Neurosci 29:9918-9929. CrossRef Medline

Liang JC, Wagner AD, Preston AR (2013) Content representation in the human medial temporal lobe. Cereb Cortex 23:80-96. CrossRef Medline

Maren S, Holt WG (2004) Hippocampus and Pavlovian fear conditioning in rats: muscimol infusions into the ventral, but not dorsal, hippocampus impair the acquisition of conditional freezing to an auditory conditional stimulus. Behav Neurosci 118:97-110. CrossRef Medline

Miller EK (1999) The prefrontal cortex: complex neural properties for complex behavior. Neuron 22:15-17. CrossRef Medline

Moser E, Moser MB, Andersen P (1993) Spatial learning impairment parallels the magnitude of dorsal hippocampal lesions, but is hardly present following ventral lesions. J Neurosci 13:3916-3925. Medline

Moser MB, Moser EI (1998) Functional differentiation in the hippocampus. Hippocampus 8:608-619. CrossRef Medline

Moser MB, Moser EI, Forrest E, Andersen P, Morris RG (1995) Spatial learning with a minislab in the dorsal hippocampus. Proc Natl Acad Sci U S A 92:9697-9701. CrossRef Medline

Nadel L, Hoscheidt S, Ryan LR (2013) Spatial cognition and the hippocampus: the anterior-posterior axis. J Cogn Neurosci 25:22-28. Medline

Navawongse R, Eichenbaum H (2013) Distinct pathways support rulebased memory retrieval and spatial mapping by hippocampal neurons. J Neurosci 33:1002-1013. CrossRef Medline

Piatti VC, Davies-Sala MG, Espósito MS, Mongiat LA, Trinchero MF, Schinder AF (2011) The timing for neuronal maturation in the adult hippocampus is modulated by local network activity. J Neurosci 31:7715-7728. CrossRef Medline

Pothuizen HH, Zhang WN, Jongen-Rêlo AL, Feldon J, Yee BK (2004) Dissociation of function between the dorsal and the ventral hippocampus in spatial learning abilities of the rat: a within-subject, within-task comparison of reference and working spatial memory. Eur J Neurosci 19:705-712. CrossRef Medline

Poucet B, Thinus-Blanc C, Muller RU (1994) Place cells in the ventral hippocampus of rats. Neuroreport 5:2045-2048. CrossRef Medline

Rogers JL, Hunsaker MR, Kesner RP (2006) Effects of ventral and dorsal CA1 subregional lesions on trace fear conditioning. Neurobiol Learn Mem 86:72-81. CrossRef Medline

Royer S, Sirota A, Patel J, Buzsáki G (2010) Distinct representations and theta dynamics in dorsal and ventral hippocampus. J Neurosci 30:1777-1787. CrossRef Medline

Shapiro ML, Tanila H, Eichenbaum H (1997) Cues that hippocampal place cells encode: dynamic and hierarchical representation of local and distal stimuli. Hippocampus 7:624-642. CrossRef Medline

Snyder JS, Ferrante SC, Cameron HA (2012) Late maturation of adult-born neurons in the temporal dentate gyrus. PLoS One 7:e48757. CrossRef Medline

Swanson LW (1981) A direct projection from Ammon's horn to prefrontal cortex in the rat. Brain Res 217:150-154. CrossRef Medline

Swanson LW, Wyss JM, Cowan WM (1978) An autoradiographic study of the organization of intrahippocampal association pathways in the rat. J Comp Neurol 181:681-715. CrossRef Medline

Tort AB, Komorowski RW, Manns JR, Kopell NJ, Eichenbaum H (2009) Theta-gamma coupling increases during the learning of item-context associations. Proc Natl Acad Sci U S A 106:20942-20947. CrossRef Medline

Tse D, Langston RF, Kakeyama M, Bethus I, Spooner PA, Wood ER, Witter MP, Morris RG (2007) Schemas and memory consolidation. Science 316:76-82. CrossRef Medline

Witter MP (2007) Intrinsic and extrinsic wiring of CA3: indications for connectional heterogeneity. Learn Mem 14:705-713. CrossRef Medline 